\title{
Permittivity Measurement using Borehole Radar from Single Hole
}

\author{
Shuhai Lin, Yoshinori Sanada, Toshifumi Matsuoka and Yuzuru Ashida
}

Dept. of Civil and Earth Resources Engineering, Kyoto University, Kyoto, 606-8501, Japan.

\begin{abstract}
This paper provides a method for measuring permittivity using borehole radar from single hole. First we use numerrical simulation to examine the suitability of borehole radar for measuring relative permittivity from single hole. We introduce a $2-\mathrm{D}$ pseudospectral time domain (PSTD) algorithm that allows us to model the full EM wavefield for rotationally symmetric structure. The effect of the borehole mud and the response of surrounding formation are simulated. It is found that the relative permittivity logging curve is sensitive to the formation interface of surrounding formation. The simulation results show that the measured relative permittivity is closed to the true parameters of the surrounding formation. We processed field data using a variance fractal dimension technique that can detect coherent borehole radar signal from background noise. The measured relative permittivity logging curve could be useful for identifying formation type and detecting water-bearing property.
\end{abstract}

\section{Introduction}

Borehole radar can provide high-resolution information around borehole. Many researchers have studied cross hole tomography. The cross hole tomography can provide electromagnetic wave velocity and resistivity structure between the two boreholes. But the cross hole method have a limit of the two-borehole distance. When the distance of the two boreholes is long, the receivers cannot receive EM signal. In this case the single hole method is suitable. Here we introduce permittivity measurement using borehole radar from single hole. We use two borehole radars, one is source, and another is receiver. When the source emits the electromagnetic wave, there are several types of electromagnetic wave in the borehole: the direct wave in the borehole mud, the reflected wave from borehole boundary and the refracted wave in the formation around the borehole. Here the refracted EM wave is used to measure the permittyity around the borehole. The center frequency of borehole radar is $100 \mathrm{MHz}$. In this frequency range, there are both conductive current and displacement current in the formation. Therefore both conductivity and relative permittivity have effect on the response of receiver. The relative permittivity is very important parameter in formation evaluation. Because relative permittivity is independent of the salinity and primarily has relationship with water content, borehole radar can detect the water-bearing characteristics of the surrounding formation such as fracture or fracture zone.

To improve understanding of the electromagnetic wave propagation associated with borehole radar, many researchers have performed numerical and experimental studies. The finite difference time-domain (FDTD) algorithm is a simple but powerful numerical method in solving 
Maxwell's equations. The second-order FDTD algorithm requires a relatively large number of nodes (usually 10-20 nodes per minimum wavelength) to achieve reasonably good accuracy. This can be attributed to its relatively large dispersion error that accumulates rapidly with time.

In contract to the FDTD algorithm which uses local approximation to the spatial derivatives, the PSTD algorithm can achieve the exact spatial derivatives with a grid density of only two nodes per minimum wavelength through the fast Fourier transform (FFT) algorithm. For borehole radar simulation, a cylindrical coordinate formulation is more pertinent because the discretization is more conformable to a borehole environment. In this paper, we present PSTD solution of Maxwell's equations in cylindrical coordinate for modeling of borehole radar. This approach allows us to explore the effects of various complicated factors on phase and amplitude of borehole radar in the presence of the borehole. In what follows, we will first present the formulation and discretization scheme, and then apply the algorithm to the simulation of some typical models.

\section{Methodology}

Here we introduce a simplified 2-D PSTD algorithm for rotationally symmetric coordinate. We use the transverse magnetic (TM) mode in which there are only $E_{r}, E_{z}$ and $H_{\phi}$. As an example, we write out the $E_{r}$ formula:

$$
E_{r}^{n+1}(i, j)=\frac{1-\frac{\sigma \cdot \Delta t}{2 \varepsilon}}{1+\frac{\sigma \cdot \Delta t}{2 \varepsilon}} E_{r}^{n}(i, j)-\frac{\frac{\Delta t}{\varepsilon}}{1+\frac{\sigma \cdot \Delta t}{2 \varepsilon}} \frac{\partial H_{\phi}}{\partial Z}
$$

The calculation of the spatial derivative can be achieved as:

$$
\frac{\partial \mathrm{H}_{\phi}}{\partial \mathrm{Z}}=F_{z}^{-1}\left\{i k_{z} F_{z}\left[\mathrm{H}_{\phi}\right]\right\}
$$

$F_{z}$ and $F_{z}^{-1}$ denote the forward and inverse Fourier transforms that are achieved by an FFT algorithm, $k_{z}$ is the wavenumber. The PSTD algorithm represents the derivative by a trigonometric polynomial 


$$
\left[\frac{\partial H_{\phi}}{\partial z}\right]_{z=z_{k}}=\frac{1}{N} \sum_{n=0}^{N-1} j n k_{z} \exp \left(\frac{j n k_{z}}{2}\right) \tilde{H}_{\phi} \cdot \exp \left(\frac{j 2 \pi m n}{N}\right)
$$

Here $\tilde{H}_{\phi}$ is the forward Fourier transforms of $H_{\phi}$. The new spectral differential operator is purely real at the Nyqiuist wavenumber, so the numerical computed derivative is also purely real. Using the same manner, we can get $E_{z}$ and $H_{\phi}$ formulas in the TM mode.

The dispersion analysis and stability condition of the 2-D PSTD algorithm can be formulated for homogeneous, lossless medium. For 2-D PSTD algorithm in cylindrical coordinates, the stability condition is given by

$$
\Delta t \leq \frac{1}{\alpha \cdot C_{\max } \sqrt{\frac{1}{(\Delta r)^{2}}+\frac{1}{(\Delta z)^{2}}}}
$$

where $\Delta r, \Delta z$ and $\Delta t$ are discretization intervals with regard to $r, z$ and $t$, respectively, $C_{\max }$ is the maximum phase velocity in finite-difference grid, $\alpha=\pi / 2 \approx 1.5708$.

\section{Numerical tests}

In the simulation the borehole radar tool contains one transmitter and several receivers. We used an ideal electric dipole as the radar source. The borehole radar is shown in Fig. 1. The solid dot denotes source and the hollow dots denote receivers. The source wavelet is the Ricker wavelet with a center frequency of $100 \mathrm{MHz}$. The source function is added to the $E_{z}$ component at each time step. Receivers are also aligned along the borehole and record the axial electric field component $E_{z}$. The borehole diameter equals $12 \mathrm{~cm}$. We simulated the electromagnetic wave propagation in the formation containing three layers.

The first example is shown in Fig. 2. The formation around borehole contains three layers with two horizontal interfaces. The parameters are shown in Table 1. The source and receivers are closed to the boundary of the borehole. 
Table 1. The parameters of example 1.

\begin{tabular}{|l|c|c|}
\hline & $\begin{array}{c}\text { Conductivity } \\
(\mathrm{s} / \mathrm{m})\end{array}$ & $\begin{array}{l}\text { Relative } \\
\text { permittivity }\end{array}$ \\
\hline Layer 1 & 0.005 & 8 \\
\hline Layer 2 & 0.03 & 20 \\
\hline Layer 3 & 0.01 & 12 \\
\hline Mud & 0.1 & 30 \\
\hline
\end{tabular}

Fig. 2(b) shows the simulated response. The waveforms show strong reflection wave from the interface of the formation because both the conductivities and the relative permittivities are different in the layer 1 and layer 2. In Fig. 2(b), the electric field can be expressed as $e^{-j k r} / r$, where $r$ is the distance between source and receive; $k$ is the wavenumber given by

$$
k^{2}=\mu \varepsilon \omega^{2}-j \mu \sigma \omega
$$

where $\omega, \mu$, and $\varepsilon$ are the angular frequency, the magnetic permeability and the dielectric permittivity, respectively.

Since $k$ is complex, it is written, as

$$
k=\alpha-i \beta
$$

$\alpha, \beta$ are given by

$$
\begin{aligned}
& \alpha=\omega\left\{\frac{\mu \varepsilon}{2}\left[\left(1+\frac{\sigma^{2}}{\varepsilon^{2} \omega^{2}}\right)^{\frac{1}{2}}+1\right]\right\}^{\frac{1}{2}} \\
& \beta=\omega\left\{\frac{\mu \varepsilon}{2}\left[\left(1+\frac{\sigma^{2}}{\varepsilon^{2} \omega^{2}}\right)^{\frac{1}{2}}-1\right]\right\}^{\frac{1}{2}}
\end{aligned}
$$

We define phase difference as

$$
\phi_{i+1}-\phi_{i}=\omega \cdot\left(T_{i+1}-T_{i}\right)
$$

where $\phi_{i}$ denotes the phase of $i$ th receiver, $T_{i}$ denotes the travel time from the source to $i$ th receiver. Fig. 2(c) and (d) show the phase difference curve and relative permittivity curve, respectively. We can see the two curves are sensitive to the interface and the measured relative 
permittivity is very closed to the true value of the formation. We found using the phase difference curve and relative permittivity curve, the formation can be identified clearly. Usually in the water saturated layer there is large relative permittivity. Therefore the water-saturated layer can be detected by using borehole radar from single hole.

The second example is shown in Fig. 3. The structure of the model is same as example 1. But the source and receivers are set in the borehole mud. Fig. 3(b) shows the waveforms have week reflection wave from the interface of the formation because the EM wave has large attenuation in the conductive mud. Fig. 3(c) and (d) show the phase difference curve and relative permittivity curve that are also sensitive to the interfases of the formation. We can see that measured relative permittivity is near to the true parameters of the formation. Comparing Fig. 2 and Fig. 3, we found the borehole mud reduces the resolution of the logging curve, but the formation can be identified clearly also.

\section{Application to field data set}

The field experiment is done at OYO Tsukuba Technology Center. Fig. 4 shows the botehole in this experiment, its diameter is $75 \mathrm{~cm}$. In the experiment we used two-borehole radar: the source and the receiver. The source diameter is $70 \mathrm{~mm}$; the receiver diameter is $37 \mathrm{~mm}$. The borehole radar center frequency is $100 \mathrm{MHz}$. The arrangement of the two borehole radars in the borehole is shown in Fig. 5. In the experiment, the source is fixed, the receiver is moving up 0.2 $\mathrm{cm}$ at each time and then records the signal. Because there is noise in the receiver signal, we use a variance fractal dimension technique to process the field data. The window size of 48 samples and the window interval of 8 sample intervals were chosen to calculate the fractal dimension values. Fig. 6 shows the sharp transition features corresponding to the first arrival of the borehole radar signal. This technique provides us with a robust and powerful tool for detecting coherent borehole radar signal from background noise. Fig. 7 shows the variables in processing borehole radar data. $Z_{i}(i=1,6)$ is the depth of the receiver, $T_{i}(i=1,6)$ is the arrive time of radar signal, $V_{i}(I=1,5)$ is the velocity of EM wave. There are such relationships:

$$
\begin{aligned}
& \left(Z_{3}-Z_{2}\right) \frac{1}{V_{2}}+\left(Z_{2}-Z_{1}\right) \frac{1}{V_{1}}=\Delta T_{31} \\
& \left(Z_{4}-Z_{3}\right) \frac{1}{V_{3}}+\left(Z_{3}-Z_{2}\right) \frac{1}{V_{2}}=\Delta T_{42} \\
& \left(Z_{5}-Z_{4}\right) \frac{1}{V_{4}}+\left(Z_{4}-Z_{3}\right) \frac{1}{V_{3}}=\Delta T_{53}
\end{aligned}
$$




$$
\left(\begin{array}{ccccc}
\Delta Z_{1} & \Delta Z_{2} & 0 & 0 & 0 \\
0 & \Delta Z_{2} & \Delta Z_{3} & 0 & 0 \\
0 & 0 & \Delta Z_{3} & 0 & 0 \\
& \cdots \cdots & & &
\end{array}\right)\left(\begin{array}{c}
1 / V_{1} \\
1 / V_{2} \\
1 / V_{3} \\
\vdots \\
\end{array}\right.
$$

where $\Delta Z_{i}=Z_{i+1}-Z_{i},(i=1,5), \Delta T_{i}=T_{i+1}-T_{i},(i=1,5)$. We can describe the problem into liner travel time inversion $A x=y$. Here $\mathrm{A}$ is a matrix, $\mathrm{x}$ is vector of the slowness of EM wave, and $y$ is the travel time difference. Fig. 8 shows the result after processing field data. We can see the measured relative permittivity can be used to identify formation, which is proven by the borehole date base (Fig. 9). The relative permittivity logging curve can provide us the information of formation type and water-bearing property.

\section{Conclusion}

Measuring permittivity from single hole using borehole radar is introduced in this paper. We have presented a PSTD solution of Mawell's equations in axial symmetric cylindrical coordinate that is suitable for modeling borehole radar. In this study, the PSTD algorithm was used to simulate the EM waveform radiated from a vertical electric dipole emitter located in the borehole filled with water-based mud. The major borehole effects on radar measurement are attenuation in conductive mud. We can observe stronger signal by setting the source and receivers along the boundary of the borehole. The simulation results show that the borehole radar introduced here can be used for EM logging and the measured relative permittivity are closed to true parameters of the surrounding formation. Using the phase difference curve and relative permittivity curve, we can identify the formation clearly. The measured relative permittivity logging curve could be useful for identifying formation type and detecting water-bearing property.

\section{Reference}

1 Ashida, Y., Murayama, Y.,and Sassa, K. (1991) Simulation of seismic disturbances by use of the Fourier Transform -model studies and considerations-, BUTSURI-TANSA, 44, 113-125.

2. Chew, W. C. (1998) Modeling of the dielectric-logging tool at high frequencies: Theory, IEEE Trans. Geosci. Remote Sensing, vol. 26, pp.383-387.

3. Chen, Y. H and Oristaglio. M. L. (2002) A modeling study of borehole radar for oil-field applications, Geophysics, 67, 1486-1494

4. Matsuoka, T., Sanada, Y., Yamamoto, K., and Ashida, Y. (1999). Inversion of EM tomography by gradient method, Proc. Of the Sos. of professional well log analysts the fifth well logging symposium of Japan, Paper J. 
5. Sanada, Y. (2000) Study on high precision modeling and imaging of the Ground Penetration Radar (GPR), Doctor thesis, Kyoto University.

6. Tada, R. (2000) Detection of first break using a variance fractal dimension technique, Proceedings of 103th SEGJ conference, 40-44.

7. Yee, K. S. (1966) Numerical solution of initial boundary value problems involving Maxwell's equations in isotropic media, IEEE Trans. Antennas Propagat., AP-14, 302-307.

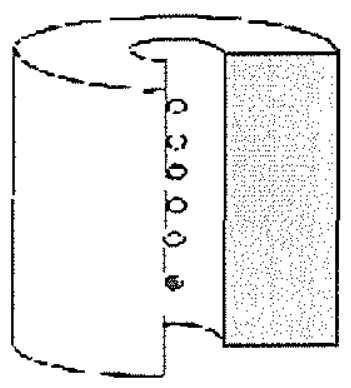

Fig. 1 Source and receiver array in the borehole.

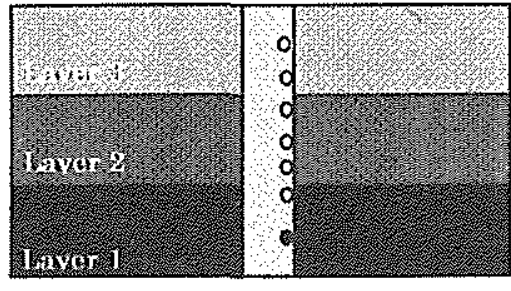

Model

(a)

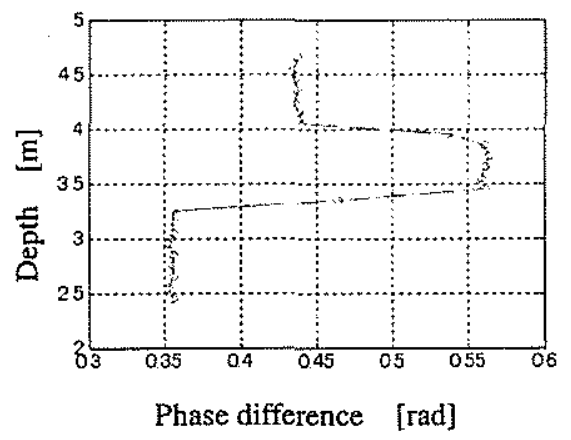

(c)

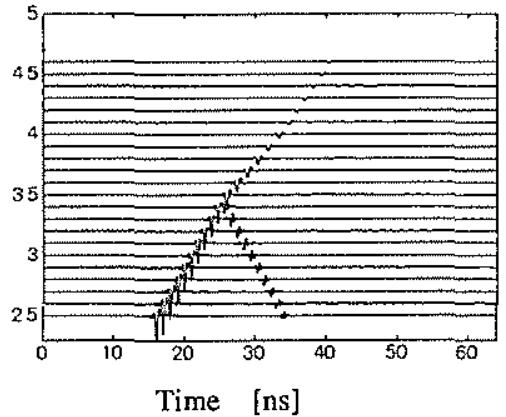

(b)

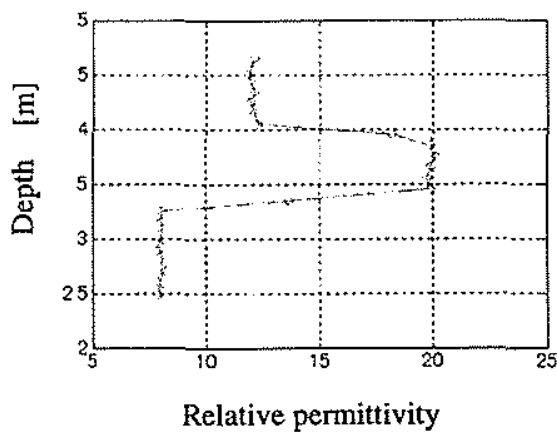

(d)

Fig. 2 Simulated results of example 1. The source and receiver are set along the boundary of the borehole. (a) Model. (b) Simulated response. (c) Phase difference. (d) Relative permittivity. 


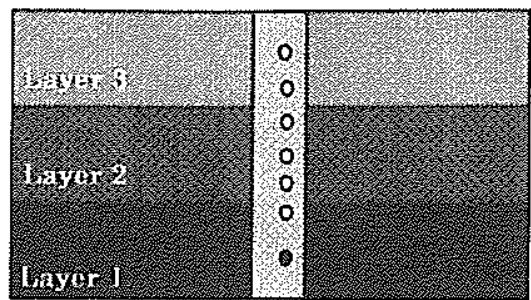

Model

(a)

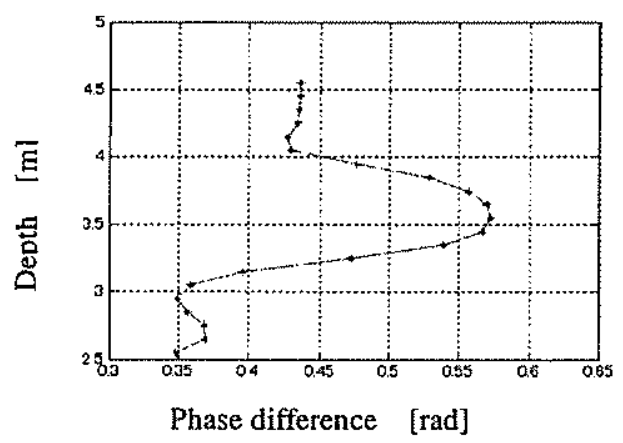

(c)

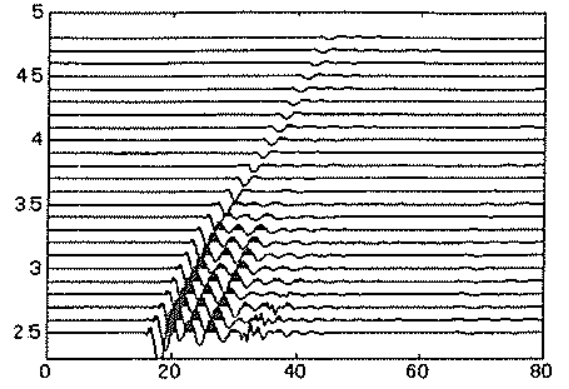

Time [ns]

(b)

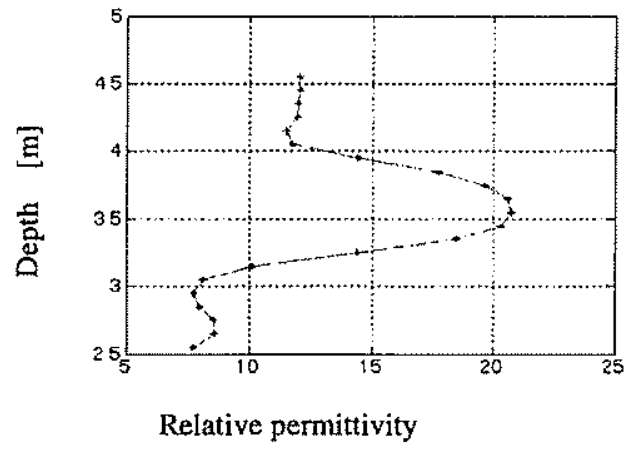

(d)

Fig. 3 Simulated results of example 2. The source and receiver are set in the borehole mud. (a) Model. (b) Simulated response. (c)Phase difference. (d) Relative permittivity.

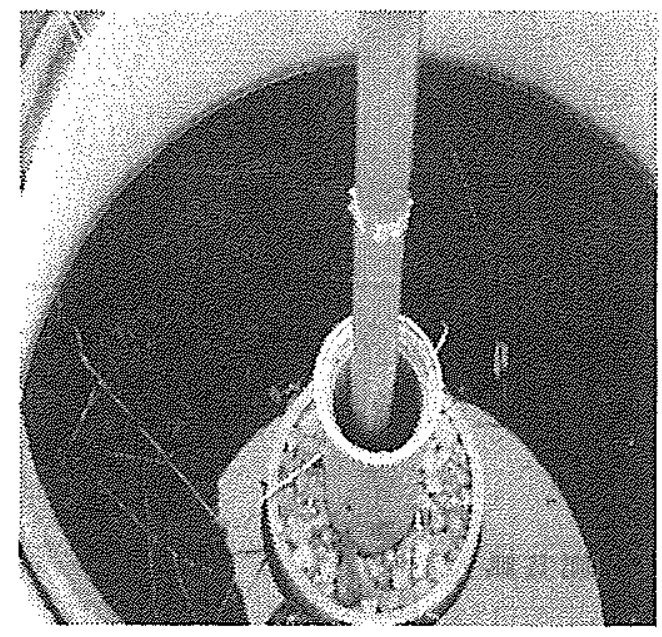

Fig. 4 The borehole in the experiment.

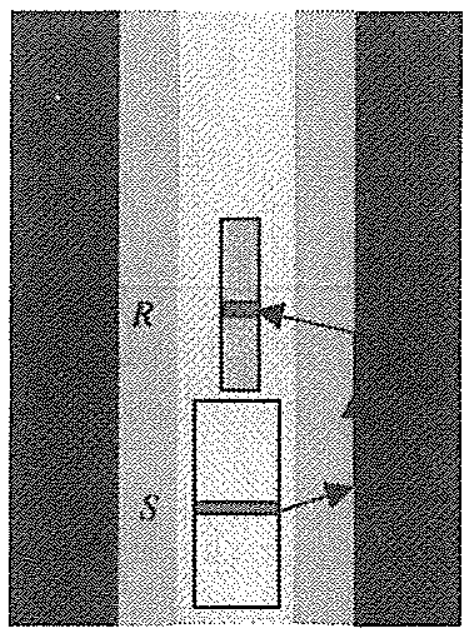

Fig. 5 The arrangement of two borehole radars. 

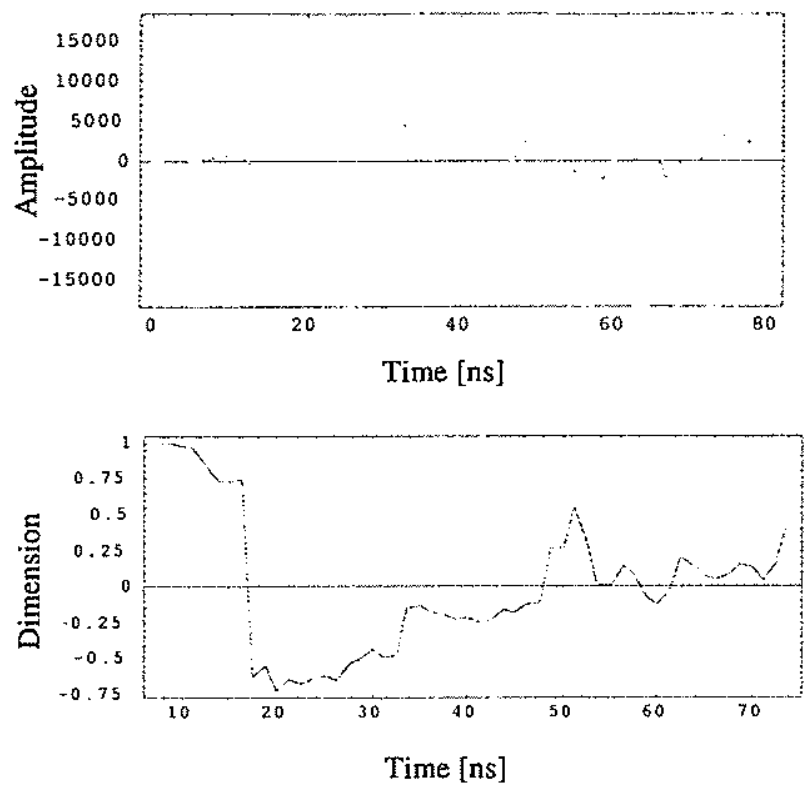

Fig. 6 Variance fractal dimension trajectory for the borehole radar signal.

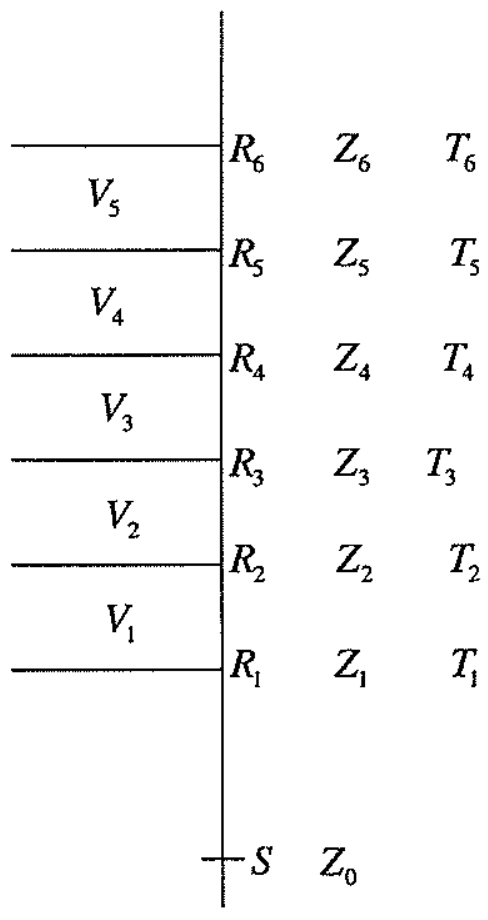

Fig. 7 Schematic illustration of formation information. 


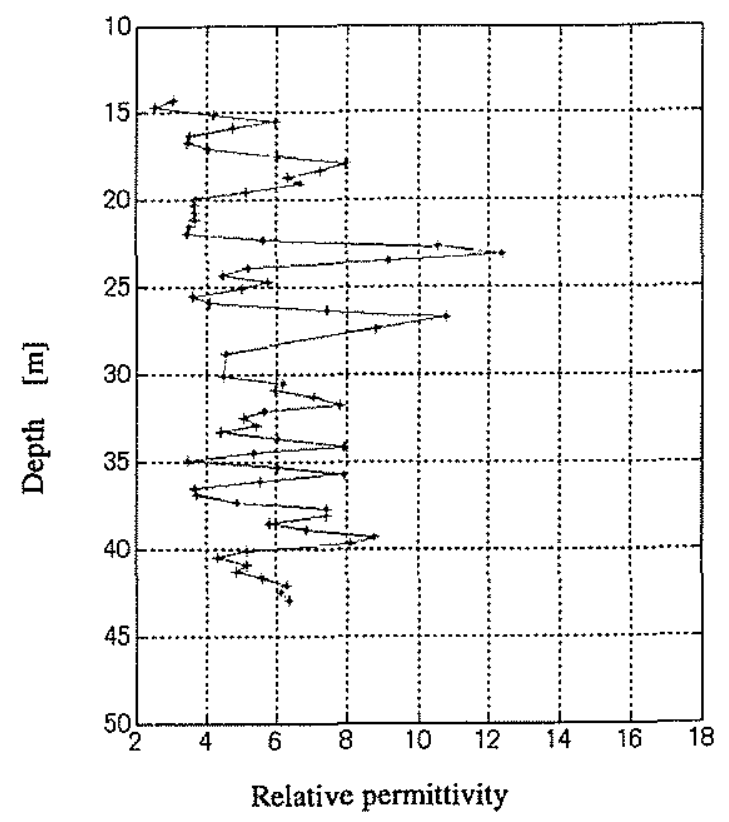

Fig. 8 The measured relative permittivity

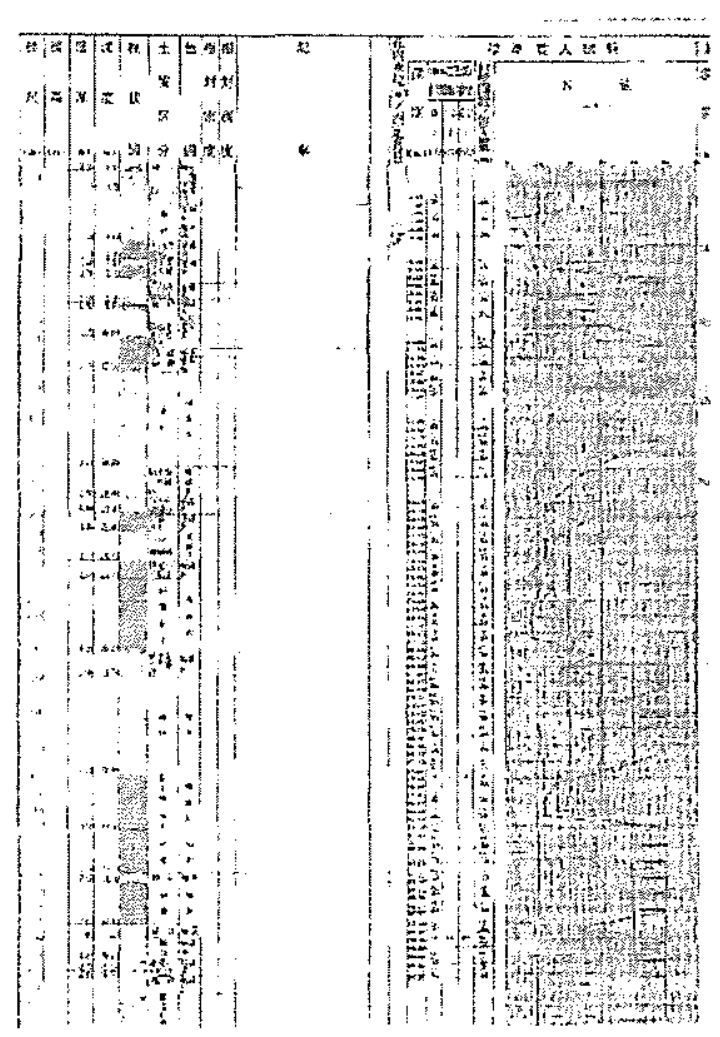

Fig. 9 The borehole data base. 